\title{
ARTICLE
}

\section{Electron density in the solar corona from type III radio bursts}

Batbayar Batmunkh and Batmunkh Damdin*

Department of Astrophysics, Institute of Astronomy \& Geophysics, Mongolian Academy of Sciences, Ulaanbaatar, Mongolia.

ARTICLE INFO: Received: 28 May, 2020; Accepted: 21 Sep, 2020

Abstract: It has been proven that electron density in the solar corona is determined by observing the frequency drift of type III radio bursts. We investigated the certain dependence of the scale height on the distance, which allows us to obtain different dependence of the frequency drift rate (FDR) on the frequency. The scale height is presented in a combination of two distance dependencies as $\mathrm{H}=$ $\alpha \lambda_{\mathrm{T}}+(1-\alpha) \mathrm{f}(r)$. As a result of integration of equation, we obtain the electron density $\mathrm{n}_{\mathrm{e}}(r)$ in the form $\mathrm{n}_{\mathrm{e}}=\mathrm{n}_{0}(1+\mathrm{y})^{-1 /(1-\alpha) \mathrm{b}}, y=\frac{1-\alpha}{\alpha} b \frac{r}{\lambda_{T}}$ and the constants are determined in comparison with the empirical formulas for FDR. In particular, using the well-known empirical formula $d v / d t=$ $-0.01 v^{1.84}$, we can obtain $(1-\alpha) \mathrm{b}=0.42$. The obtained calculation results are compared with the results of other authors and they have been found to be consistent when choosing the parameters included in the formula. The calculation shows that this formula is in good agreement with the data at distances from the lower corona to the Earth's orbit. This dependence of electron density makes it possible to agree with the observed FDR as a function of frequency.

Keywords: Sun: corona; Sun: electron density; Sun: radio burst type III; Sun: frequency drift rate;

\section{INTRODUCTION}

The electron density in the solar corona is an important physical parameter for studying the active phenomena of the solar atmosphere. In [1] it was written that' Indeed, the profile of the Sun's electron number density with radial distance $r$ from the Sun's center, ne(r), is poorly understood and measured from the photosphere, where the medium is almost entirely neutral and cool $(\sim 6000 \mathrm{~K})$, to the fully ionized and hot plasma of the corona $\left(10^{6}\right.$ $\left.10^{7} \mathrm{~K}\right)$ and solar wind $\left(10^{4}-10^{6} \mathrm{~K}\right) "$. Therefore, due to these different physical conditions for the altitude in the solar atmosphere, electron density does not change merely owing to distance. Many works are have been devoted to determining the electron density in the solar corona from various observational data depending on altitude [1-8].

\footnotetext{
*corresponding author: batmunkh.damdin@yahoo.com
}

https://orcid.org/0000-0002-9990-0898

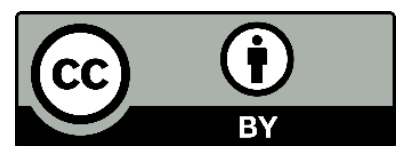

The Author(s). 2020 Open access This article is distributed under the terms of the Creative Commons Attribution 4.0 International License (https://creativecommons.org/licenses/by/4.0/), which permits unrestricted use, distribution, and reproduction in any medium, provided you give appropriate credit to the original author(s) and the source, provide a link to the Creative Commons license, and indicate if changes were made. 
For electron density, scientists have used exponential expressions near the base of the solar corona and $a_{i} / r^{b_{i}}$ type sum with some coefficients away from the Sun, as well as their combinations. Using observational data, average values of unknown coefficients are determined, that is, they are adjusted to these data.

The most striking manifestation of the sporadic radio emission of the Sun are the type III radio bursts. Solar radio bursts were observed for almost 70 years, and one of the first observational data was described in detail in [9]. Since the electron density in the solar corona decreases with height, frequency drift is observed for radio bursts. Radiation of burst types (III, V, and U) are generated at frequencies close to the Langmuir and double Langmuir frequencies (second harmonic) corresponding to the electron density at this height. From plasma theory, the Langmuir frequency (fundamental emission) is defined as $v \approx 9000 \sqrt{n_{e}}, \mathrm{~Hz}$. Where $n_{e}$ (in cgs units) is the background electron density at the location of the exciter. Currently, there are several theories for generating type III bursts and the most prominent among them are the works by Ginzburg and Zheleznyakov [10], and Melrose $[11,12]$. On the way through the corona, a fast

\section{MATERIALS AND METHODS}

\section{Density Calculations}

From the formula $v \approx 9000 \sqrt{n_{e}}$, it is possible to determine electron density from the observed frequencies, however, the distance of radiation or the radiation source from the center of the Sun is unknown $\left(n_{e}(r)-\right.$ this dependence on radial distance is very important for studying the physical states of the corona). That is $n_{e} \approx 1.24 \times 10^{-8} f^{2}$ for fundamental and $n_{e} \approx 3.10 \times 10^{-9} f^{2}$ for harmonic emission [7]. For many problems, an explicit expression of the electron density as a function of the height and the temperature is required. Aschwanden and Acton [23] showed that electron density in the corona varies significantly, depending on the position of the angle at a given height. For example, the smallest values are measured in coronal hole electron beam excites plasma turbulence, the radiation of which we observe in the form of radio bursts. An electron beam that generates type III bursts propagates along open field lines through the corona. The radiation source moves in the solar corona at a speed of $0.1 \mathrm{c}$ to $0.8 \mathrm{c}$. Typical velocities are between 0.2 and $0.5 \mathrm{c}$ [13].

From spectral observations, the drift frequencies of radio bursts are calculated, and there are also several empirical formulas for the FDR that correspond to different frequency ranges [14-20]. They are obtained in the form $\frac{d v}{d t}=-A v^{\varepsilon}$ with various coefficients (A) and powers $(\varepsilon>1)$. It can be seen from the hydrostatic equilibrium in the corona that the FDR is obtained in the form $d v / d t=-A v$, that is, a linear frequency dependence $(\varepsilon=1)$. Therefore, as noted in [21], the dependency of the FDR on frequency vary significantly between empirical and theoretical formulas.

In this paper, we obtained a formula for determining the electron density in the solar corona, taking into account the change in the scale height $\lambda_{T}=47.0[\mathrm{Mm}] \times T_{h}[\mathrm{MK}]$ [22] with altitude, and the constants are determined by way of comparison with empirical formulas for FDR

regions, of order $n_{0} \approx(0.8-1.1) \times 10^{8} \mathrm{~cm}^{-3}$ and the highest densities are found in active regions (AR), of order $n_{0} \approx(0.4-2.0) \times$ $10^{9} \mathrm{~cm}^{-3}$. Many works $[1-3,5-8]$ show that the dependence of electron density on the height differs from hydrostatic equilibrium $(P=\rho g H)$ with a constant scale height $\mathrm{H}$ (from the exponential density model). It was found that the coronal streamers and ARs have a scale height that exceeds the hydrostatic scale height by a factor of up to 2.3 [23,24]. In our analysis, a function $f(z)$ with a weight $(1-\alpha)$ that is directly proportional to the distance was added to the scale height $\left(\alpha \lambda_{T}\right)$ in the equation of hydrostatic equilibrium. The equation reads as follows: 


$$
\begin{aligned}
& H=\alpha \lambda_{T}+(1-\alpha) f(z), \quad 0<\alpha \leq 1, \\
& d p=-\rho g d z, \quad \rho=p / g H, \\
& \frac{d p}{p}=-\frac{d z}{\alpha \lambda_{T}+(1-\alpha) f(z)} .
\end{aligned}
$$

If $f(z)=b z$ then the result of the integration in $z$ is

$$
P=P_{0}\left[(1+y)^{1 / y}\right]^{-h / \alpha \lambda_{T}}, \quad y=\frac{1-\alpha}{\alpha} b \frac{h}{\lambda_{T}} .
$$

A similar result, the electron density $\left(n_{e}=0.52 P / k T\right.$ [19] $)$ will be

$$
n_{e}=n_{0}\left[(1+y)^{1 / y}\right]^{-h / \alpha \lambda_{T}}
$$

And

$$
n_{e}=n_{0}(1+y)^{-1 /(1-\alpha) b} .
$$

The limiting value of this goes to hydrostatic equilibrium (barometric equation)

$$
\lim _{\alpha \rightarrow 1} n_{e}=n_{0} \lim _{y \rightarrow 0}\left[(1+y)^{1 / y}\right]^{-h / \alpha \lambda_{T}} \rightarrow n_{0} e^{-\frac{h}{\lambda_{T}}} .
$$

In the case of a constant $\lambda_{T}$ for $\partial n_{e} / \partial h$ we get the following:

$$
\frac{\partial n_{e}}{\partial h}=-\frac{n_{e}}{\alpha \lambda_{T}(1+y)}
$$

The constants $\alpha$ and $b$ will be determined by comparison with empirical formulas for FDR.

\section{a) Frequency drift rate}

It is known that the frequency drift rate is determined in terms of electron density $n_{e}$ in the solar corona. The drift rate can be written as a PDE [21,22]:

$$
\frac{d v}{d t}=\frac{\partial v}{\partial n_{e}} \frac{\partial n_{e}}{\partial h} \cos \phi \frac{\partial s}{\partial t}
$$

where $\phi$ is the angle between the beam direction and the vertical $(\partial h)$.

Equation (4) has to be corrected for the finite speed of light. The differential in observing time, $\partial t$, is shortened by the relativistic motion of the source along its $\partial s$ [21], $\partial t=\partial s / v_{s}-$ $(\partial s \cos \theta) / v_{g r}, \theta$ is the angle between the beam direction and the radiation path to the observer, $v_{s}$ is the source velocity, $v_{g r}$ is the group velocity of the radiation.

As a result of the differentiation of the corresponding parameters in (4) and $n_{e}=$ $n_{0} \exp \left(-h / \lambda_{T}\right)$, the following were obtained [21]:

$$
d v / d t=-A v, \quad A=\frac{v_{s} \cos \phi}{2 \lambda_{T}(1-\beta \cos \theta)}
$$


$v_{g r} \approx c, \quad \beta=v_{s} / c$. On the other hand, the empirical relation is represented as $d v / d t=$ $-0.01 v^{1.84}$ [14]. The obvious discrepancy of this linear dependence on frequency with the empirical relation can have several reasons [21]: (i) the source velocity $\left(v_{S}\right)$ may decrease with altitude, (ii) the scale height $\lambda_{T}$ may increase with altitude.

In many works [25-27] it has been noted that the source velocity is almost constant when passing through the corona and is at $v<$ $14 \mathrm{MHz}$ begins to decrease. In [27] a deceleration of $-12.3 \pm 0.8 \mathrm{~km} \mathrm{~s}^{-2}$ was suggested. In [28], it was noted that the change in velocity as a function of distance is very small. Therefore, in all likelihood, the scale height varies with respect to distance. We will pay attention to this point. Put (3) in (4), and formula (5) will take the following form for FDR:

$$
\begin{gathered}
\frac{d v}{d t}=-\frac{v W}{\alpha \lambda_{T}+(1-\alpha) b h}, \\
W=\frac{v_{s} \cos \phi}{2(1-\beta \cos \theta)} .
\end{gathered}
$$

If we express FDR in terms of frequency, taking into account (3), then we would have

$$
\frac{d v}{d t}=-v\left(\frac{v}{v_{0}}\right)^{2(1-\alpha) b} \frac{W}{\alpha \lambda_{T}}
$$

So we got $d v / d t \sim-v^{2(1-\alpha) b+1}$, which can have different degrees.

\section{b) Finding the constants $b$ and $\alpha$}

Empirical formulas can be used to determine the constants $b$ and $\alpha$. There are several empirical formulas for FDR [14-20] and one of them is $d v / d t=-0.01 v^{1.84}$ (denote $I$ )
[14], which many authors refer to. Comparing this with expression (7), we can define constants $b$ and $\alpha$. For example, if we use an empirical expression for FDR as $A v^{\varepsilon}$, then

$$
A v^{\varepsilon} \equiv v\left(\frac{v}{v_{0}}\right)^{2(1-\alpha) b} \frac{W}{\alpha \lambda_{T}}
$$

This comparison shows that the degrees of frequency in the right and left sides of the equality should be equal to $2(1-\alpha) b+1=\varepsilon$. From (8) we determine the temperature of the solar corona, which is obtained by $T_{h}=$ $W /\left(47.0 \times A \times v_{0}^{\varepsilon-1} \times \alpha\right)$, where $v_{0}[\mathrm{MHz}]=$ $0.009 \sqrt{n_{0}}$ and $n_{0}$ is the electron density at the coronal base. It is clear that $\alpha \lambda_{T}=$ const $\times W$.

\section{RESULTS AND DISCUSSION}

It is known that solar corona is heterogeneous both in the whole space and in time, including the electron density. Most empirical formulas for the electron density are determined from observable data reflecting the dependence on the distance; therefore, some physical parameters are not explicitly included
If the temperature of the solar corona is known, then from this formula we can determine the value of the parameter $\alpha$. Alternatively, the choice of $\alpha$ determines the temperature. Therefore, at a known temperature of the corona, the constants $\alpha$ and $b$ are uniquely determined. in the formula. In formulas $(2,6,7)$, the scaleheight temperature $T_{h}$ of the medium enters explicitly through $\lambda_{T}$. It was shown in the work [29] that the electron kinetic temperature $T_{e}$ in the corona is substantially less than $T_{h}$. In our case, we did not take into account this difference $\left(T_{h}=T_{e}\right)$. We proposed a scale 
height depending on distance in a first approximation as in (1). In [30], the free optical thickness of the radiating medium is giving by the formula

$$
\tau \approx 1.5 \times 10^{-6} T_{e}^{-3 / 2} v^{2} H \sim T_{e}^{-3 / 2} n_{e} H .
$$

The radiation coming to us leaves the layer of the solar corona with an optical depth of less than unity. For a given frequency, the optical thickness is constant and, therefore, $n_{e} H$ does not change much taking into account the temperature dependence on the distance. This allows us to represent the scale heights in the form (1).

In the work [7], radial distributions of the mean temperature in the streamer, calculated according to the data from EIS, are presented along with their comparison with the data of other authors [31, 32]. Here the temperature is in the range $1 \mathrm{MK}-1.5 \mathrm{MK}$ with deviations from the mean value of about $1.43 \mathrm{MK}$. In the works [26, 33], a formula $T_{e}=0.37 \times$
$10^{-4} v^{4 / 3} t_{d}^{2 / 3}$ is given for determining the coronal electron temperature from the observed time profile of type III bursts. Alvarez and Haddock [34] in the frequency range $200 \mathrm{MHz}$ to $50 \mathrm{kHz}$ found a decay time that is approximated $t_{d}=10^{7.7} v^{-0.95}$, where $t_{d}$ is in seconds and $v$ is in Hz. Using these values, we can obtain an approximation $T_{e}[M K] \approx 0.08 \times$ $v^{0.7}$ for the coronal electron temperature in frequency, where $v$ is in $\mathrm{MHz}$.

Fig. 1 shows the dependence of the scale heights $(H / \tau)$ calculated for various optical thickness and temperatures. At constant temperature $H / \tau \approx h$, then the scale height increases steeply with height. In all likelihood, the behavior of all $H / \tau$ is similar, and especially when the temperature dependence is taken into account, they are identical. Therefore, in a first approximation, expression (1) for the scale heights can be adopted.

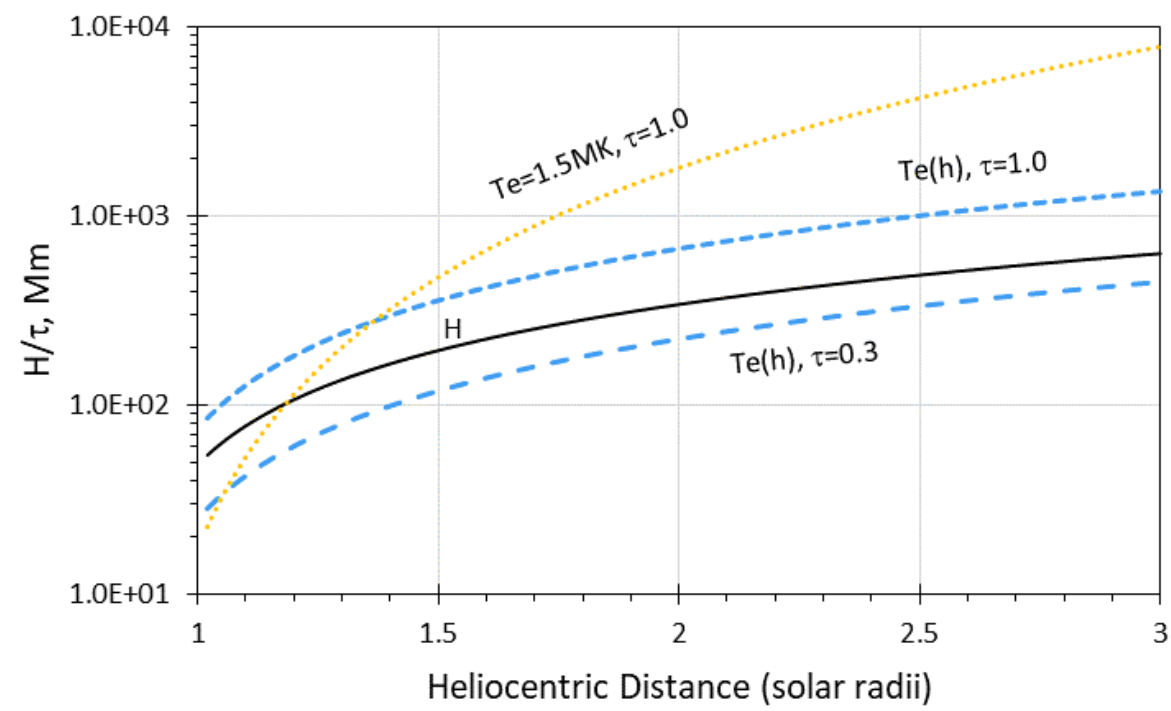

Figure 1. Scale heights $(H / \tau)$ calculated for various optical thicknesses and temperatures (blue dotted, dashed and solid black lines). $T_{e}(v)=0.08 \times v^{0.7}$

Also $H / \tau$ were calculated for constant temperature (yellow dotted line).

The expression in (2) for electron density can be written in another form as $n_{e}=$ $n_{0}(1+d / \mu \cdot h)^{-1 / d}, \mu=\alpha \lambda_{T}=$ const $\times W$, $d=\varepsilon / 2$. The coefficient $\mu$ depends only on the initial value of the electron density $\left(n_{0}\right)$. For average values of parameters, with $v_{s}=0.3$, $\cos \phi=0.5$ and $\cos \theta=0.5$, finding the constants $\mu, d$ from the comparison (8) with (I) [14], we obtain $n_{e}=n_{0}(1+0.017$. $h)^{-2.38}$. The distance $h$ is measured in Mm. (I) - the best fit for the data in the range $75 \mathrm{kHz}-$ $550 \mathrm{MHz}$, and in this interval most radio bursts are observed. The parameter $\alpha$ is $\approx$ 0.2582 at $T_{h}=2.0 \mathrm{MK}$. We can also use other empirical formulas, such as $d v / d t=$ $-0.1 v^{1.4}$ (denote $I I$ ) (High frequencies 200$3000 \mathrm{MHz}$ ) [15]. 


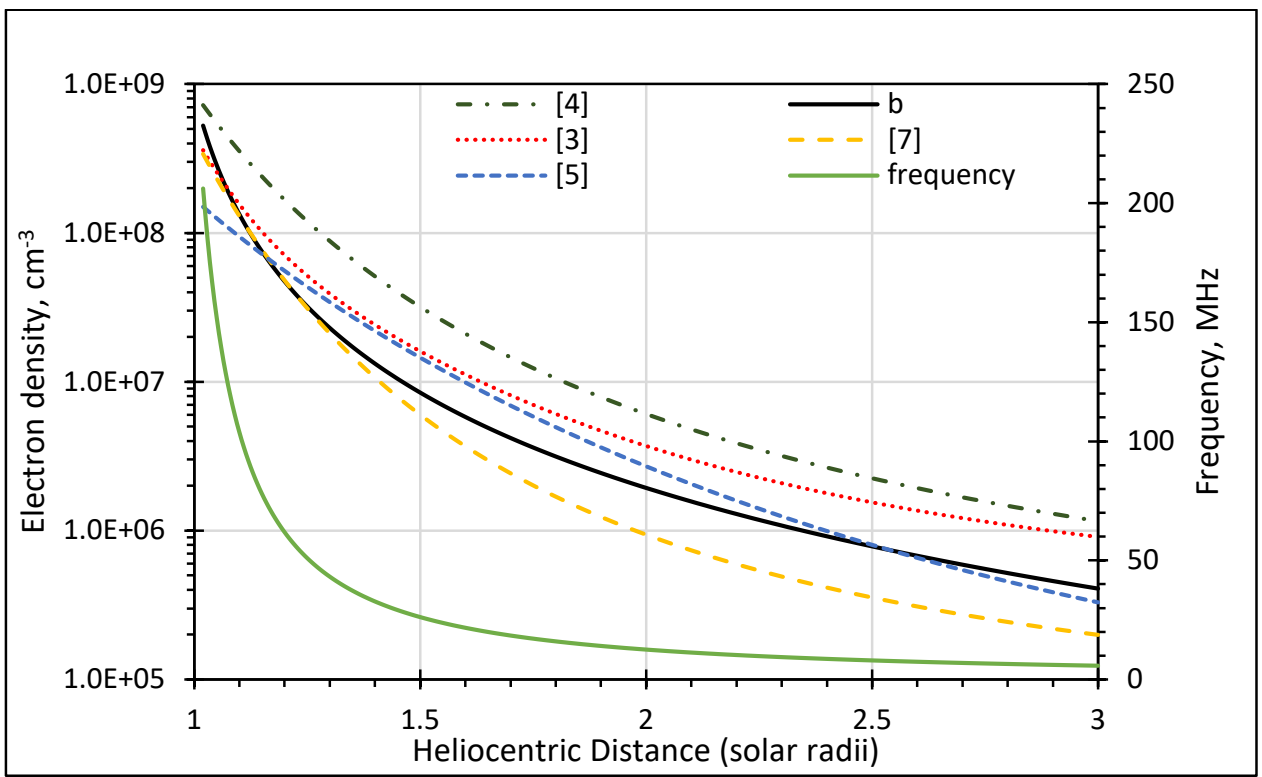

Figure 2. Given the electron density of the solar corona, calculated by various formulas [3-5,7]. $b$-are calculated by $n_{e}=n_{0}(1+0.017 \cdot h)^{-2.38}$ (black full line) and the green line is a radial dependence of the local electron frequency $v_{p e}(r) . n_{0}=8.775 \times 10^{8}[4]$.

The calculation result with the average values of the parameters of this formula are shown in Fig. 2 (black full line) and compared with the results of other authors from the literature. In general terms, these results are consistent with each other. Our results can fluctuate within the range of changes in the value $v_{p}=v_{s} \cos \phi$. Bougeret et al. [35] derived a radial density of heliosphere by employing in-situ density measurements. The best fit of the data are obtained by $n_{r}=6.14$. $\left(r / R_{1 A U}\right)^{-2.10}$ [35]. Robinson [36] deduced a radial density of $n_{r} \approx 7.0 \cdot\left(r / R_{1 A U}\right)^{-2.29}$. Figure 3 shows a comparison of these with the formula (2) at a distance from the corona to the Earth's orbit. It can be seen that the results are in good agreement with each other. In the Earth's orbit, they give values of 6.14 [35], 7.0 [36] and $7.3 \mathrm{~cm}^{-3}[\mathrm{~b}]$, respectively.

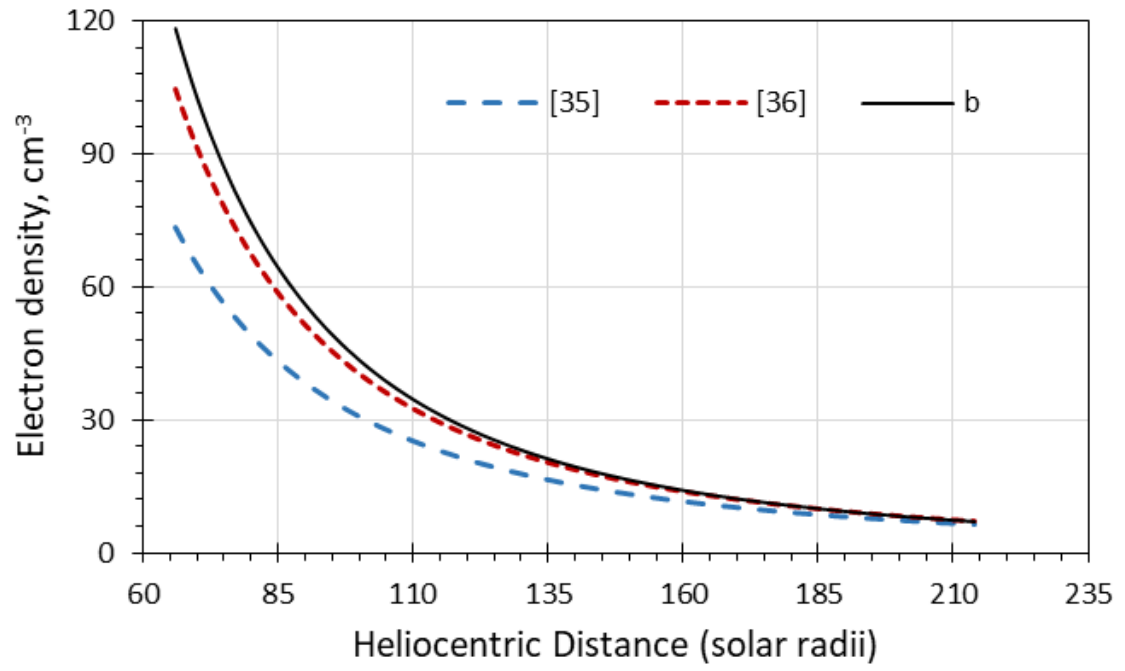

Figure 3. Comparison of the radial density from [35.36] with the electron density calculated by the formula (2) at a distance of the heliosphere (to Earth's orbit). 


\section{CONCLUSIONS}

An expression is obtained for the electron density of the solar corona, which allows to obtain consistent results between the theoretical and empirical formulas for the FDR of type III radio bursts. The main idea is that the scale height $(H)$ in the lower corona is almost constant and that at large distances from the base of the corona it changes in proportion to the distance. In the general case, $(\mathrm{H})$ can be represented by more complex functions leading to a more complicated integration. From the above formula for the electron density of the corona, a general expression for the FDR was obtained as $d v / d t \sim-v^{2(1-\alpha) b+1}$. In particular, for $\alpha=1$ it reduces to the barometric law, for $2(1-\alpha) b+1=1.84$ to the formula (I) and other (II). The numerical values of formula (2) directly depend on the

\section{REFERENCES}

1. Cairns, I.H., Lobzin, V.V., Warmuth, A., Li B., Robinson, P.A., Mann, G., (2009). "Direct radio probing and interpretation of the sun's plasma density profile", Astrophys. J. Lett., Vol. 706, pp. 265-269.

2. Baumbach, S., (1937). "Strahlung, Ergiebigkeit und Elektronendichte der Sonnenkorona”, Astronomische nachrichten, Vol. 263, Issue 6, pp.121-134.

3. Allen, C.W., (1947). "Interpretation of electron densities from corona brightness", MNRAS, Vol. 107, pp. 426-432.

4. Newkirk, G.Jr., (1961). "The Solar corona in active regions and the thermal origin of the slowly varying component of solar radio radiation”, ApJ, Vol.133, pp. 9831013.

5. Saito, K., Poland, A.I., \& Minro, R.H., (1977). "A study of the background corona near solar minimum”, Sol. Phys., Vol. 55, pp.121-134.

6. Leblanc, Y., Dulk, G.A., \& Bougeret, J.-L., (1998). "Tracing the electron density from the corona to 1au”, Sol. Phys., Vol. 183, pp.165-180. projection $v_{p}\left(v_{p}=v_{s} \cos \phi\right)$ of the source velocity. In the case of a type III radio burst, the direction of the source velocity is mainly along the line of the corona's open magnetic field (probably $\cos \phi \sim 1.0$ ). It can be seen from figures 2 and 3 that formula (2) is in good agreement with the data at distances from the lower corona to the Earth's orbit. Finally, we note that formula (2) is not empirical, that is, it was obtained under a certain assumption and, therefore, it can be transformed in various ways for specific problems.

Acknowledgments This work was funded by the Science and Technology Foundation of Mongolia (Project No. 2018/30). We are very grateful to Dr. Chraibi Mohsine for the valuable comments and suggestions.

7. Goryaev, F., Slemzin, V., Vainshtein, L. \& Williams, D.R., (2014). "Study of EUV emission and properties of a coronal streamer from PROBA2/SWAP, Hinode/EIS and Mauna Loa Mk4 observations”, ApJ, Vol. 781, pp.100-134.

8. McCanley, P.I., Cairns, I.H., \& Morgan, J., (2018). "Densities probed by coronal type III radio burst imaging”, Sol. Phys., Vol. 293, pp.132-159.

9. WiId, J.P., (1950). "Observations of the spectrum of high-intensity solar radiation at metre wavelengths. III. Isolated bursts", AuSRA, Vol.3, pp. 541-557.

10. Ginzburg,V.L. \& Zheleznikov,V.V., (1958). "On the possible mechanisms of sporadic solar radio emission (radiation in an isotropic plasma)", Astronomicheskii Zhurnal, Vol. 35, pp. 694-712.

11. Melrose, D.B., (1980). "The emission mechanisms for solar radio bursts”, Space Sci. Rev., Vol. 26, pp. 3-38.

12. Melrose, D.B., (2008). "Coherent emission”, IAU Symposium, Vol. 257, pp. 305-315. 
13. Reid, H.A.S. \& Kontar, E.P., (2018). "Spatial expansion and speeds of type III electron beam sources in the solar corona", ApJ, Vol. 867, pp. 158-173.

14. Alvarez, H. \& Haddock, F.T., (1973). "Solar wind density model from km-wave type III bursts”, Sol. Phys., Vol. 29, pp. 197-209.

15. Aschwanden, M.J. et al., (1995). "Solar electron beams detected in hard X-rays and radio waves”, ApJ, Vol. 455, pp. 347-365.

16. Melnik, V.N., Konovalenko, A.A., Rucker, H.O., et al., (2011). "Observations of powerful type III bursts in the frequency Range 10 - 30 MHz”, Sol. Phys., Vol. 269, pp. 335-350.

17. Stanislavsky, A.A., (2017). "Solar type III bursts with high-frequency cut-off", Astron. Nachr., Vol. 338, pp. 407-412.

18. Ratcliffe, H., Kontar, E.P., Reid, H.A.S., (2014). "Large scale simulations of solar type III radio bursts: flux density, drift rate, duration and bandwidth”, A\&A, Vol. 572, A111, pp. 1-11.

19. Mann, G., Jansen, F., MacDowall, R.J., Kaiser, M.L., \& Stone, R.G., (1999). “A heliospheric density model and type III radio bursts”, A\&A, Vol. 348, pp. 614620.

20. Zhang, P.J.; Wang, C.B.; Ye, L., (2018). "A type III radio burst automatic analysis system and statistic results for a half solar cycle with Nançay Decameter Array", A\&A, Vol. 618, pp. A165,1-11.

21. Benz, A.O., (2002). "Plasma astrophysics", Kluwer academic publishers, pp. 107-109.

22. Aschwanden, M.J., (2004). "Physics of the solar corona, hydrostatics”, Springer, pp. 67-69.

23. Aschwanden, M.J. \& Acton, L.W., (2001). "Temperature tomography of the soft Xray corona: Measurements of electron densities, temperatures, and differential emission measure distributions above the limb”, ApJ, Vol. 550, pp. 475-492.

24. Klassen, A., Karlick'y, M. \& Mann, G., (2003). "Superluminal apparent velocities of relativistic electron beams in the solar corona”, A\&A, Vol. 410, pp. 307-314.
25. WiId, J.P. \& Smerd, S.F., (1972). "Radio bursts from the solar corona”, Ann. Rev. Astron.Astrophys. Vol. 10, pp. 159-196.

26. Elgaroy, O. \& Lyngstad, E., (1972). "Highresolution observations of type III solar radio bursts”, A\&A, Vol. 16, pp. 1-12.

27. Krupar, V., Kontar, E. P., Soucek, J., et al., (2015). "On the speed and acceleration of electron beams triggering interplanetary type III radio bursts”, A\&A, Vol. 580, A137, pp. 1-6.

28. Reid, H.A.S. \& Kontar, E.P., (2018). "Solar type III radio burst time characteristics at LOFAR frequencies and the implications for electron beam transport”, A\&A, Vol. 614, pp. A69, 1-15.

29. Mercier, C. \& Chambe, G., (2015). "Electron density and temperature in the solar corona from multifrequency radio imaging”, A\&A, Vol. 583, pp. A101, 1-11.

30. Dulk, G.A., (1985). "Radio emission from the Sun and stars", Ann. Rev. Astron.Astrophys. Vol. 23, pp. 169-224.

31. Gibson, S.E., Fludra, A., Bagenal, F., Biesecker, D., Del Zanna, G., \& Bromage, B., (1999). "Solar minimum streamer densities and temperatures using Whole Sun Month coordinated data sets", Geophys. Res., Vol. 104, pp. 9691-9699.

32. Vasquez, A.M., van Ballegooijen, A. A., \& Raymond, J. C., (2003). "The effect of proton temperature anisotropy on the solar minimum corona and wind”, ApJ, Vol. 598, pp. 1361-1374.

33. Hughes, M.P. \& Harkness, R.L., (1963). "Spectral observations of solar radio bursts. IV. The exciters of fast-drift bursts”, ApJ, Vol. 138, pp. 239-251.

34. Alvarez, H. \& Haddock, F.T., (1973). "Decay time of type III solar bursts observed at kilometric wavelengths”, Sol. Phys., Vol. 30, pp.175-182.

35. Bougered, J.-L., King, J.H. \& Schwenn, R., (1983). "Solar radio burst and in-situ determination of interplanetary electron density”, Sol. Phys., Vol. 90, pp. 401-412.

36. Robinson, P.A., (1992). "Radial variation of type III source parameters”, Sol. Phys., Vol. 137, pp. 307-315. 